\title{
Journal of Health \& Medical Informatics
}

\section{Design of a Smartphone Application with Integrated Functional Electrical Stimulation (FES) Treatment Randomization and On-The-Fly Stimulus Parameter Adjustment for Streamlining the Clinical Evaluation of FES Protocols}

\author{
Sweeney $\mathbf{D}^{1}$, Corley $\mathbf{G} \mathbf{J}^{1}$, Browne $\mathrm{P}^{2,3}$, Burridge $\mathbf{J H}^{4}$, Quinlan $\mathbf{L R}^{2,5^{*}}$ and ÓLaighin $\mathbf{G}^{1,5}$ \\ ${ }^{1}$ Electrical and Electronic Engineering, School of Engineering and Informatics, NUI, Galway, Ireland \\ ${ }^{2}$ Physiology Department, School of Medicine, NUI Galway, Ireland \\ ${ }^{3}$ Neurology Department University Hospital Galway, Ireland \\ ${ }^{4}$ Facility of Health Sciences, University of Southampton, UK \\ ${ }^{5}$ CÚRAM Centre for Research in Medical Devices, NUI Galway, Ireland
}

*Corresponding author: Leo Quinlan, NUI Galway, University Road, Galway, Ireland, Tel: 00353 91493710; E-mail: leo.quinlan@nuigalway.ie

Received date: December $5^{\text {th }}, 2015$; Accepted date: March $7^{\text {th }}, 2016$; Published date: March $14^{\text {th }}, 2016$

Copyright: $\odot 2016$ Sweeney D, et al. This is an open-access article distributed under the terms of the Creative Commons Attribution License, which permits unrestricted use, distribution, and reproduction in any medium, provided the original author and source are credited.

\begin{abstract}
The clinical application of Functional Electrical Stimulation (FES) has evolved over the last five decades. However, the use of the Randomized Control Trial (RCT) methodology in evaluating the clinical effectiveness of new and existing applications of FES is a demanding process adding time and cost to these trials. Consequently, there has been a low level of RCTs applied to FES studies. Poor quality trials result in poor evidence of FES effectiveness with a consequence that the technique may not be adopted into clinical practice. In this paper some of the key challenges encountered in FES randomised control clinical trials are identified and a solution to address these challenges is presented in the form of a smartphone App and a Bluetooth controlled FES architecture. The design and evaluation of a smartphone application using a User Centred Design approach to provide automatic blind randomization control and facilitating the wireless temporal control of a portable Bluetooth enabled FES device is described.
\end{abstract}

\section{Introduction}

\section{Functional electrical stimulation}

Functional Electrical Stimulation (FES) is the application of Neuromuscular Electrical Stimulation (NMES) in real-time (typically under sensor control) to contract a muscle to perform a functional task. FES technology has advanced significantly, particularly in the last two decades. New applications and new approaches to existing applications of FES are being continually investigated to find improved treatments for patients with a variety of clinical pathologies [1-3]. To establish the effectiveness of any new FES application or of a new approach to an existing application of FES, it must be evaluated using a robust clinical investigation.

\section{Randomized control trials}

A key requirement of an effective clinical investigation is an experimental methodology that features (i) unbiased randomization into at least two comparable groups, (ii) blinded assessment by clinicians and ideally (iii) blinding of the participants to their intervention allocation. The most commonly used and robust clinical investigation design is the Randomized Controlled Trial (RCT). A well conducted RCT is regarded as the gold standard method for evaluating the efficacy or / and effectiveness of a health or medical treatment [4]. Although, performing a RCT effectively adds time and cost to a clinical investigation [5], failure to not adequately adhere to this methodology can result in poor clinical evidence being generated. Without robust evidence being generated through clinical evaluation, technical advances are unlikely to be adopted into routine clinical practice. Thus providing strong clinical evidence is essential so patients are not deprived access to potentially new medical treatments that could positively impact on their lives.

\section{Implementing RCTs}

Step 1 Randomization sequencing: The first step in implementing an effective RCT requires the randomization sequencing of treatments. Randomization sequencing aims to blind trial organisers and participants to the sequence of allocated intervention treatments. Treatment allocation can be performed easily by assigning each treatment randomly [6,7]. A number of websites and desktop programs are capable of providing either random number tables or random number generators running locally on a PC or hosted by an Internet server [8]. However, as identified by Saghaei et al. most of the internet services have limited capabilities with respect to the block design specification and control over the output format [8]. These services can provide randomization sequencing but they fail in the ability to provide treatment allocation concealment and assignment.

Step 2 Allocation concealment and assignment: Allocation concealment and assignment is the second step in implementing an effective RCT. Methods used to implement allocation concealment and assignment commonly use a central telephone randomization system and numbered containers $[9,10]$. Traditionally, sequentially numbered, opaque sealed envelopes (SNOSE) have been the method for 
concealment, particularly in resource-poor settings [10]. Using the SNOSE method [11], treatments are randomly concealed within opaque sealed envelopes and sequentially assigned to participants as they enrol in the trial. However, this method has been shown to be prone to tampering $[9,12]$. Treatment allocations may be revealed if envelopes are held up to a bright light or envelopes may be opened in advance of participant enrolment. As a result, some reviewers treat trials using envelopes as a means of allocation concealment with caution [13-15]. Alternatively, scratch card technology has been recently adopted. Research staff who had previously used the SNOSE system for random allocation reported the scratch card technology easier to use [16]. To date the use of scratch card technology is limited and may be due to the limited number of companies equipped to develop the technology. The use of a central systems such as $24 \mathrm{~h}$ central randomization office (telephone or web-based) offer a high level of security, with stringent procedures and trained staff members [9]. Central systems ensure allocation concealment by independently assigning treatments and withholding assignment information from research staff until all participants are assigned. Armijo-Olivo et al. showed that from 2005 to $2011,18.9 \%$ of the included trials used central randomization, $21.6 \%$ used SNOSE, and $13.5 \%$ used unsafe envelopes and $46 \%$ used non-safe methods of allocation concealment [17]. In a review of RCT published in 2011, Clark et al. [18] highlighted that, $15 \%$ of the included trials used central randomization while $31 \%$ used envelopes as a method of allocation concealment. In contrast, Chen et al. [19] reported that between 2011 to 2013 the number of trials using central randomization / pharmacy surpassed that of sealed envelopes.

Step 3 Allocation of assigned intervention: Following allocation concealment and assignment, a member of the research staff allocates the assigned intervention treatment. Intervention allocation is the third step in implementing an effective RCT and is performed by an investigator which is typically an independent doctor, clinician or researcher. Intervention allocation is highly dependent on the design of each trial. Typical, during an investigator blinded RCT with a SNOSE method of assignment, a member of the research staff would open the participant's assigned envelope and provide the investigator with the assigned intervention. Alternatively, if a central randomization method of assignment is used, a member of the research staff would receive a participant's treatment from the central randomization provider via telephone, email or fax, and provide the investigator with the assigned intervention. To ensure the investigator cannot decipher the assigned intervention treatment, treatments are typical undisguisable from each other. During a double blind RCT, the participant and investigator is unaware of the intervention received although this is not possible in all cases due to the type of treatment. In general the implementation of an RCT varies between medical disciplines due to the different types of intervention and trial methodologies. The techniques used to provide randomization, allocation concealment assignment and intervention allocation are adjusted to each trial needs with the aim.

\section{Functional electrical stimulation trial methodologies}

Designing a protocol for an RCT using an FES device is particularly challenging as there is a wide range of parameters that can be adjusted in any FES treatment (Figure 2): pulse width, inter-pulse interval, pulse frequency, ramp-up time, ramp-down time, ON-time and OFF-time and different stimulus intensity envelope shapes. An FES trial may want to assess the impact of changing these parameters using a variety of permutations and combinations. For example, over the period 2014-2015, in a search of the PubMed, Scopus, Science Direct and
Google Scholar databases, 23 studies were identified where FES protocols were investigated with multiple FES treatments. All publications reported using randomization but only 1 publication reported the method of randomization. De et al. reported each treatment was randomly chosen for each participant using Randomizer v4.0 software [20]. Four publications reported using single blinding and two used double blinding. Within the reported double blinded studies, Dantas et al. [21] reported the blinding of both investigator and participant to the treatment being applied, but did not specify how this was achieved and Scot et al. [22] reported the blinding of both observer (individual who viewed the treatment effects) and participant to the treatment being applied, but again did not specify how this was achieved. None of the papers identified referred to implementing allocation concealment and assignment.

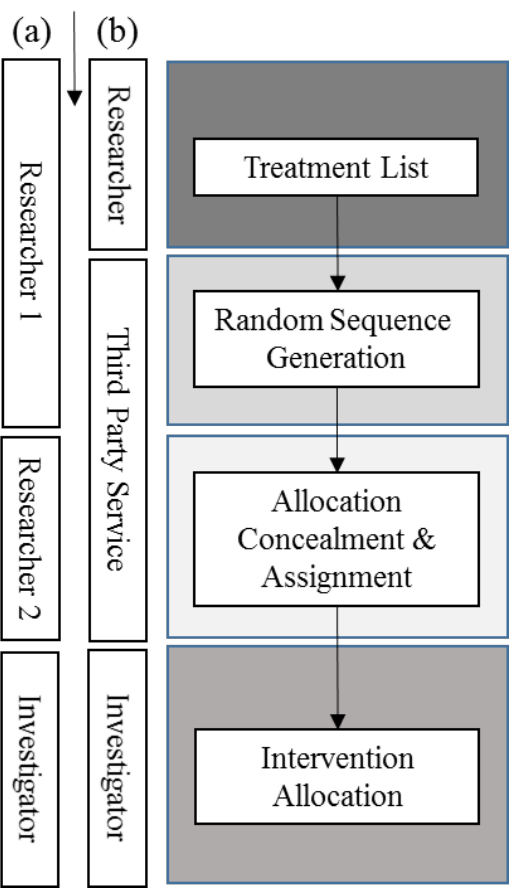

Figure 1: General RCT randomization procedure: (a) without third party services (i.e., SNOSE) (b) with third party services (i.e., Central randomisation office).

Although the above identified studies report using randomization they lack the methodological requirements for an RCT, and as such only one identified study classified itself as an RTC [21]. It is clear that the implementation of an RCT methodology (Figure 1) is very demanding as it involves both extensive time and cost to the trial. A high level of discipline and adherence to the protocol is required from the research staff. Extensive manual data collection and handling requires strict quality assurance and control. An additional challenge in FES trials is the need to manually configure the FES device for each participant, prior to their treatment; this is highly time consuming and is prone to error. The manually configuration effectively eliminates blinding during the intervention allocation process unless the manual adjustments are achieved through an independent investigator. 


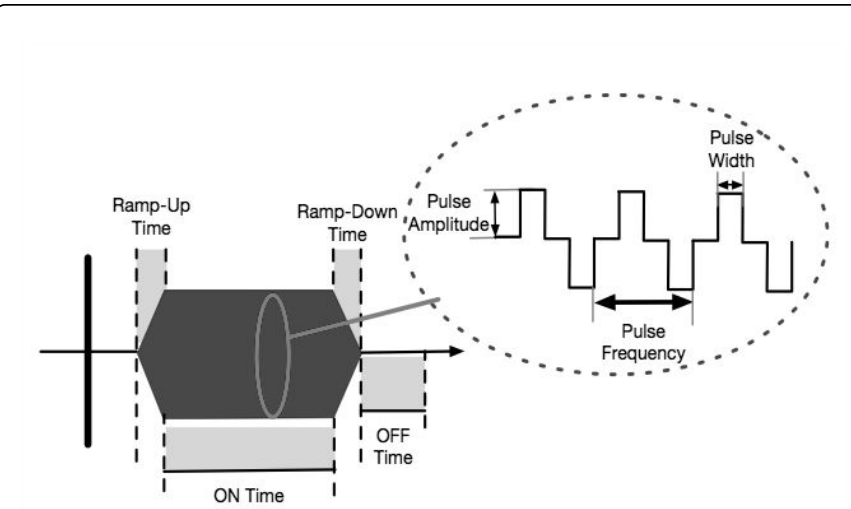

Figure 2: Common adjustable FES treatment parameters.

In a randomized sham control study of direct current stimulation, $\mathrm{Wu}$ et al. streamlined the manual configuration of their stimulation device, by programming the device to automatically generate an active or sham device state according to the parity of a random number. The random number generated was inputted into the device by an independent investigator [23]. In another trial Szecsi et al. [24] enabled the configuration of a stimulation device through a physical switch which selected between two pre-programmed stimulation treatments [25]. While these approaches reduced the time to configure the stimulation devices by loading pre-configured treatments, they still required an independent investigator to configure the device to match the participant's allocated treatment.

An additional developing challenge in FES trials is the temporal (on-the-fly) control of stimulus. For participant-worn FES devices the manual temporal control of stimulus delivery by the clinician in realtime can be difficult. Manual temporal control typically requires a wired connection from a clinician control switch to a sensor / control input on the stimulator device, if it is available. To date temporal control of FES is typical achieved through tethered FES systems $[26,27]$. The usability of such tethered FES systems in clinical settings can be an undesirable factor influencing the trial's outcome. The temporal control of an FES treatment by the clinician, while the participant is, for example, walking, is highly cumbersome.

The work presented in this paper is focused on addressing these identified challenges associated with using FES in clinical trials. We propose a solution to address these challenges through the adoption of smartphone technology. The design of a smartphone application providing automatic blind randomization control functionality and facilitating the wireless temporal control of a portable Bluetooth enabled FES device is described.

\section{Methods}

\section{Design requirements}

In order to design an effective smartphone App in conjunction with a Bluetooth-enabled portable FES device, certain specific design requirements were required to be met. These requirements were identified in response to the identified challenges in using FES in clinical trials. Primarily it was required to meet the core functional requirement for treatment randomization and temporal wireless control and also it was required to provide a good user experience for clinicians and researchers. Ethical approval to test the system was granted by University Hospital Galway research ethics committee.

\section{Blind randomization control requirements}

Developing an App that has the ability to transparently provide treatment allocation concealment, randomization and blind allocation assignment was a key design challenge. The App was required to facilitate the evaluation of repeated measures (within participant) RCTs. The FES parameters; Stimulus Intensity, Frequency, ON / OFF time, and Ramp-up / down time formed the parameters of treatment. To allow the generation of treatments, a GUI was necessary to enable adjustment of FES parameter values. A key system requirement was the use of robust randomization technique, as insufficient randomization could produce biased results, which would compromise the reliability of the App. Additionally, to control possible treatment order effects, treatment order balancing was required. Blinding of both the participant and the clinician from the selected treatment required the automatic configuration of the FES device stimulus parameters. To enable data analysis, all treatment parameters and participant data were required to be logged on the smartphone.

\section{Wireless temporal stimulator control requirements}

Wireless Temporal Stimulator Control allows the wireless control of a Bluetooth ${ }^{1}$ enabled FES device in real-time. Currently the IEEE 11073 Personal Health standard, which addresses the interoperability of personal health devices, has no device specialization standard for FES devices. As such a specific Bluetooth proprietary communication protocol was required, which incorporated a precise design to ensure a reliable protocol with low latency. To facilitate the adjustment of the FES device parameters by a clinician, including the control of treatment delivery, a custom GUI was designed. Additionally, safeguards were required to eliminate malicious or accidental user misuse. Such safeguards were incorporated through tactile and visual feedback during critical operations. Additionally, it was critical that unauthorized receivers cannot intercept any wirelessly transmitted data, which could promote tampering. To allow the safe delivery of a controlled stimulus treatment, delivery was required to be implemented in a manner that only permits delivery under the direct control of the clinician.

\section{Usability requirements}

The proposed App was intended for research use by clinicians and researchers assessing FES treatment strategies. To achieve this, the App interface was required to be ergonomic in design, allowing young and older users to operate the App without difficulty. For small-screen devices where the user's attention is at a premium, the efficiency by which the user can adjust the control settings was an extremely important design consideration [28]. To reduce the complexity of the interface the user was often only required to access a minimum control set. Therefore, it was proposed that the interface should be kept both narrow (minimum choice on screen) and shallow (minimum screen hierarchy levels) enabling the users to complete actions as quickly as possible.

$1 \quad$ Bluetooth SIG Inc., 5209 Lake Washington Blvd NE, Suite 350, Kirkland, WA 98033, USA. 
Citation: Sweeney D, Corley GJ, Browne P, Burridge JH, Quinlan LR, Laighin ÓG (2016) Design of a Smartphone Application with Integrated Functional Electrical Stimulation (FES) Treatment Randomization and On-The-Fly Stimulus Parameter Adjustment for Streamlining the Clinical Evaluation of FES Protocols. J Health Med Inform 7: 220. doi:10.4172/2157-7420.1000220

Page 4 of 9

\section{System Overview}

The system consisted of a smartphone, a mobile App and a Bluetooth compatible FES device. The architecture of the purposed system is presented in Figure 3 which highlights the main components of the system. The Motorola Moto G smartphone manufactured by MotorolaTM was selected as the system smartphone. The Moto G provides useful features for the application such as a 4.5 " LCD touch screen, Bluetooth low energy (BLE) connectivity, internal 16 GB storage and Google AndroidTM 4.3 Operating System (OS) preinstalled. Android OS is an open source platform offering a high level of software control over its device features, permitting this commercially available device to be used as a research tool.

The App, developed in $\mathrm{Java}^{2}$, for the Android OS platform, implemented Android's Application Program Interface (API) to employ the smartphones built-in functions and functionality. The App was developed for 4.3 or later Android OS, which supported the BLE network and was tested and debugged using Android StudioTM version $0.8 .6^{2}$.

The FES device utilized for the system was a research device designed in-house at NUI Galway. BLE wireless technology was incorporated into the FES design to facilitate the wireless control and optimization of stimulation parameters. The FES device Bluetooth GATT profile established common operations and a framework for data transmission, defining the attribute protocol for discovering services, and reading/writing characteristic values on the FES device GATT server.

\section{Blinded randomized control overview}

The blind randomization control requirements were such that the clinician could manually generate FES treatments, based on the selection of treatment parameter values. Adjustment values ranged from Stimulus Intensity ( $0 \%-100 \%$ in increments of $1 \%)$, Frequency $(30 \mathrm{~Hz}-40 \mathrm{~Hz}$ in increments of $1 \mathrm{~Hz}), \mathrm{ON} / \mathrm{OFF}$ time $(0 \mathrm{~s}-2 \mathrm{~s}$ in increments of $0.2 \mathrm{~s})$ and Ramp - up / down time ( $0 \mathrm{~s}-2 \mathrm{~s}$ in increments of $0.2 \mathrm{~s}$ ). A maximum of 100 treatments could be created by saving multiple treatments in an Android "List" class. Each new treatment entered was added to the Treatment List " $T_{n+1}$ ", where $n$ is the number of treatments in the list, Figure 4.

Upon completion of the Treatment List the clinician selects the number of participants, "P". To ensure that the generated order of treatments are sequenced in a counterbalanced fashion (a balanced Latin Square [29] was implemented). To implement a balanced Latin Square protocol, the number of participants " $P$ " enrolled must be a multiple of the number of treatments $\mathrm{T}_{\mathrm{n}}$ generated, therefore $P=n \times$ $m$, where $\mathrm{m}$ is a multiplier. The balanced Latin square was implemented using an array "L" of size $n \times n$, with elements $1_{(i, j)}$ representing a treatment $\mathrm{T}_{\mathrm{n}}$, in which $(l(i, j), l(i, j+1))$ elements pairs were all distinct. To facilitate the selection of multiple participants and treatment blocks " $b$ ", L was duplicated " $\mathrm{m} \times \mathrm{b}$ " times resulting in a Treatment Array "TA" of size $(n \times m) \times(n \times b)$, Figure 5 .

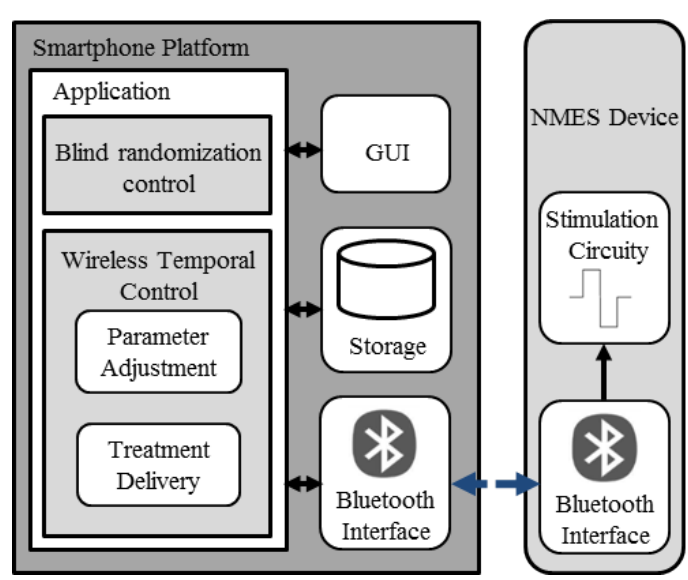

Figure 3: Proposed system architecture.

Each row " $i$ " of matrix TA represented a Treatment Order "TOi", which can be allocated to a participant. Loading TOi into a list and implementing the Android shuffle class, based on Fisher and Yetas algorithm [30], allowed the rows of TA to be shuffled randomly, generating a random distribution of Treatment Orders. Figure 6 illustrate the generation of a random distribution of Treatment Orders for an RCT with four Treatments and the enrolment of eight participants with two treatment blocks.

Blind allocation of the treatment orders was achieved through Androids built-in BLE API, permitting the wireless configuration of the FES device for each participant, prior to the delivery of their treatment. During the allocation process the App automatically assigned the participants sequentially with the rows " $\mathrm{i}$ " of the Treatment Array, Pi ta (i, j), Figure 7. Treatment Allocations were stored in the internal memory of the smartphone and only become visible to the clinician after the trial was completed, eliminating possible biasing during testing.

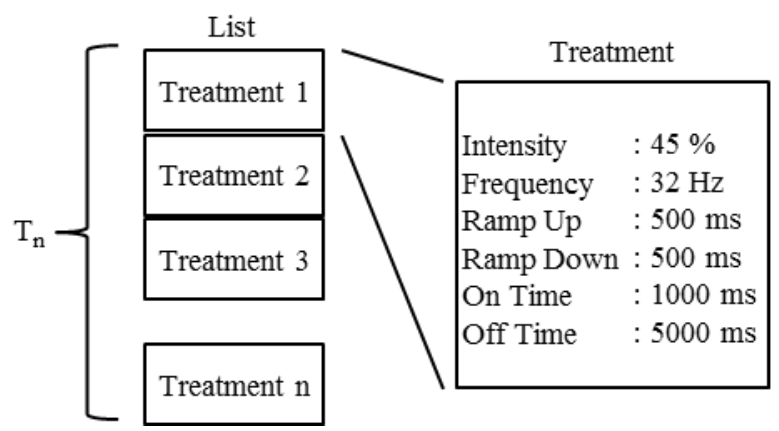

Figure 4: Treatment list structure. 
Citation: Sweeney D, Corley GJ, Browne P, Burridge JH, Quinlan LR, Laighin ÓG (2016) Design of a Smartphone Application with Integrated Functional Electrical Stimulation (FES) Treatment Randomization and On-The-Fly Stimulus Parameter Adjustment for Streamlining the Clinical Evaluation of FES Protocols. J Health Med Inform 7: 220. doi:10.4172/2157-7420.1000220

Page 5 of 9

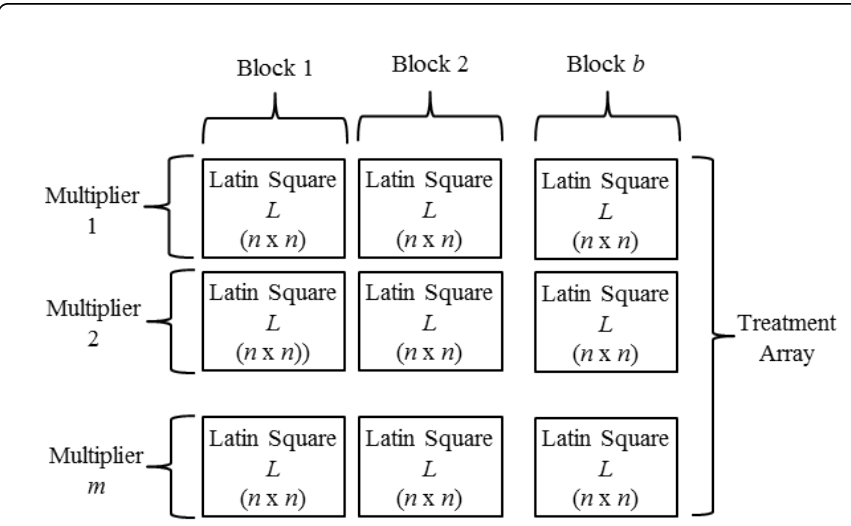

Figure 5: Treatment array structure (Unshuffled).

$$
\begin{aligned}
& L=\left[\begin{array}{cccc}
T_{1} & T_{2} & T_{4} & T_{3} \\
T_{2} & T_{3} & T_{1} & T_{4} \\
T_{3} & T_{4} & T_{2} & T_{1} \\
T_{4} & T_{1} & T_{3} & T_{2}
\end{array}\right]
\end{aligned}
$$

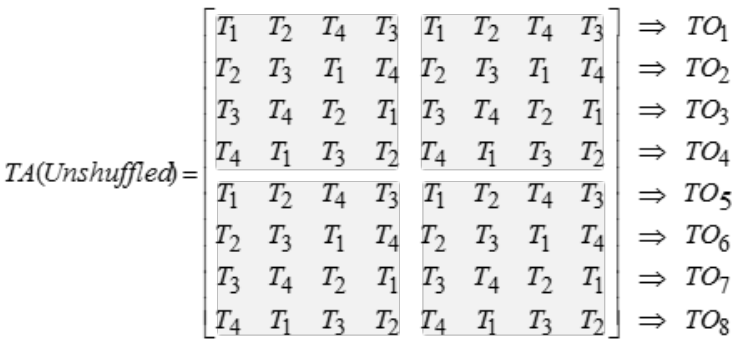

$$
\begin{aligned}
& \text { TA(Shuffled })=\left[\begin{array}{llllllll}
T_{3} & T_{4} & T_{2} & T_{1} & T_{3} & T_{4} & T_{2} & T_{1} \\
T_{1} & T_{2} & T_{4} & T_{3} & T_{1} & T_{2} & T_{4} & T_{3} \\
T_{1} & T_{2} & T_{4} & T_{3} & T_{1} & T_{2} & T_{4} & T_{3} \\
T_{4} & T_{1} & T_{3} & T_{2} & T_{4} & T_{1} & T_{3} & T_{2} \\
T_{3} & T_{4} & T_{2} & T_{1} & T_{3} & T_{4} & T_{2} & T_{1} \\
T_{4} & T_{1} & T_{3} & T_{2} & T_{4} & T_{1} & T_{3} & T_{2} \\
T_{2} & T_{3} & T_{1} & T_{4} & T_{2} & T_{3} & T_{1} & T_{4} \\
T_{2} & T_{3} & T_{1} & T_{4} & T_{2} & T_{3} & T_{1} & T_{4}
\end{array}\right] \Rightarrow T O_{3} \Rightarrow T O_{1} \Rightarrow \begin{array}{l}
\Rightarrow T O_{4} \\
\Rightarrow T O_{6} \\
\Rightarrow T O_{2}
\end{array}
\end{aligned}
$$

Figure 6: Treatment order randomization example, $\mathrm{n}=4, \mathrm{P}=8, \mathrm{~m}$ $=2, \mathrm{~b}=2$.

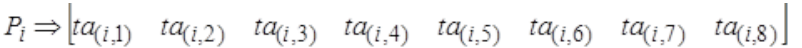

$$
\begin{aligned}
& P_{8} \Rightarrow\left[\begin{array}{llllllll}
T_{2} & T_{3} & T_{1} & T_{4} & T_{2} & T_{3} & T_{1} & T_{4}
\end{array}\right] \\
& P_{1} \Rightarrow\left[\begin{array}{llllllll}
T_{3} & T_{4} & T_{2} & T_{1} & T_{3} & T_{4} & T_{2} & T_{1}
\end{array}\right]
\end{aligned}
$$

Figure 7: Participant treatment allocation. P8 (participant eight) is allocated treatment order number 8 (row eight of the treatment array). P1 (participant one) is allocated treatment order number 1 (row one of the treatment array).

\section{Wireless temporal stimulator control overview}

Wireless temporal control provided the capability for on-the-fly FES parameter adjustment and FES treatment delivery control via the App. The BLE specification uses a client-server architecture, which enables the communication of packets using a synchronous request-response messaging pattern. This protocol ensured synchronization between the App and the FES device, with all transmitted request packets requiring the receipt of a response packet. Failure to receive a response within the time-out period resulted in the re-transmission of the request until a response was acknowledged. To ensure all transmitted data remain secure, an encryption, authentication and authorization security protocol inherits to Bluetooth was implemented. The implemented wireless temporal control enabled the clinician to: Progress through a participant's Treatment Order and transparently uploading treatments to the FES device.

Deliver treatments by pressing a single tactile on-screen button, which when pressed started a treatment and which upon release stopped the treatment.

During FES value adjustments safeguards are employed to check and verify new values were within the prescription range of the FES parameters for the patient. In the event of an invalid value the clinician was alerted by popup message on the smartphone. An automatic logging feature allowed adjustments and treatment delivery activities to be stored on the internal memory storage of the smartphone.

\section{Usability overview}

The App implemented four activity classes. The classes were coded to perform two main system functions, (i) blind randomization control and (ii) wireless temporal stimulator control. Each activity was associated with a unique GUI, Figure 8 . Activities were coded to handle clinician input through Android's standard Spinner's, Edit Text's and Buttons classes. The App consists of only two hierarchy levels. In the initial hierarchy level the user was presented with the main menu GUI, which provided the choice of three options to advance to the next hierarchy level, Figure 9. The second hierarchy level enabled the user to generate treatments, allocate treatments and control treatment delivery through individual GUIs, Figure 8.

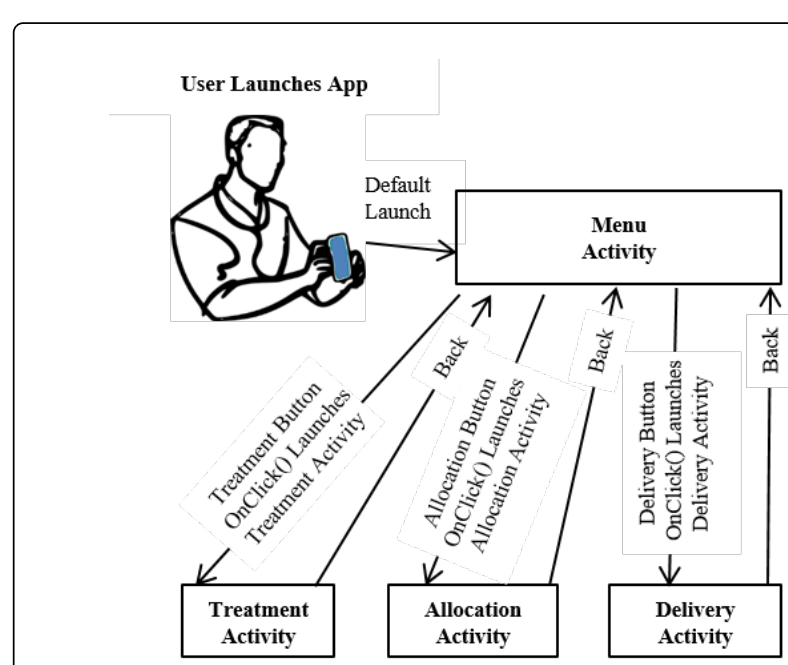

Figure 8: App activity design. 
Citation: Sweeney D, Corley GJ, Browne P, Burridge JH, Quinlan LR, Laighin ÓG (2016) Design of a Smartphone Application with Integrated Functional Electrical Stimulation (FES) Treatment Randomization and On-The-Fly Stimulus Parameter Adjustment for Streamlining the Clinical Evaluation of FES Protocols. J Health Med Inform 7: 220. doi:10.4172/2157-7420.1000220

Page 6 of 9

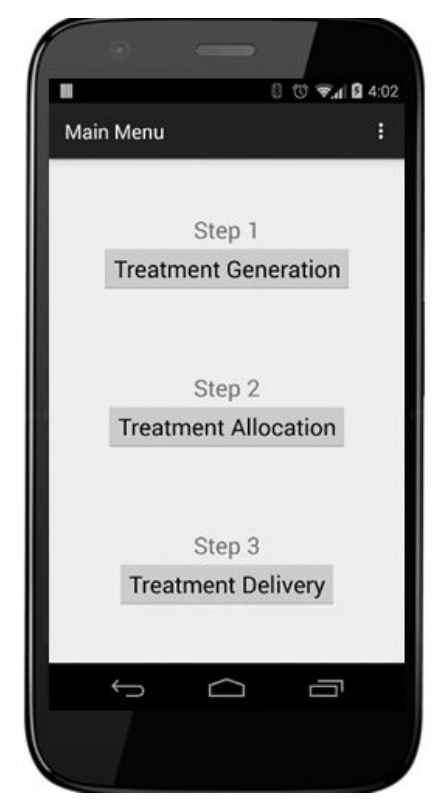

Figure 9: App screenshot of main menu.

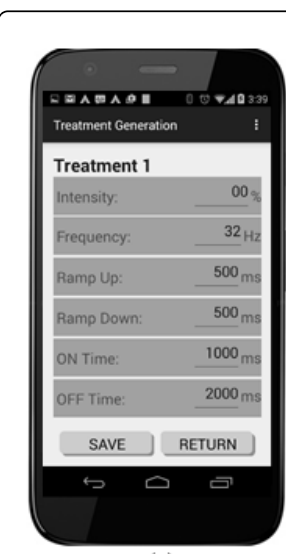

(a)

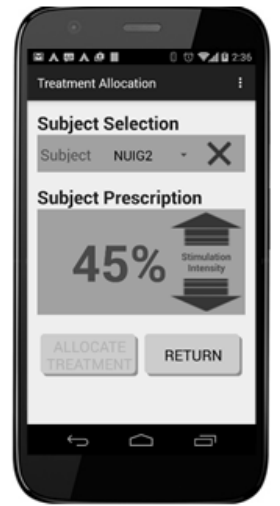

(b)

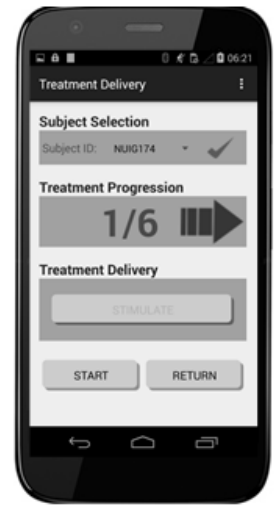

(c)
Figure 10: App Screenshot of: (a) Treatment generation GUI, (b) Treatment allocation GUI, (c) Treatment delivery GUI.

\section{Evaluation Methodology}

\section{Blinded randomized control}

The goal of the Blinded Randomized Control evaluation was to determine whether the randomization process could be implemented successfully within a smartphone application, and to assess its implemented features to ensure reliability. Evaluation was performed using statistical tools to assess if randomization was successfully achieved. The "runs test" [31] and the normal probability plot were performed using Matlab. The runs test (Wald-Wolfowitz test) is a nonparametric statistical test that checks the null hypothesis that a series of data are generated randomly Figure 10. Normal probability plots assess if data arise from a normal distribution. The process involved the simulation of an RCT with six treatments, six participants and one treatment block. The simulation was performed 7200, 72,000, 720,000 times and evaluation of the resulting series of Treatment Arrays with the "runs test" was performed. The normal probability plot assessed the frequency occurrence of each of the resulting series of Treatment Arrays.

\section{Wireless temporal stimulator control}

The goal of the Wireless Temporal Stimulator Control evaluation was to determine the reliability and performance of the App's communication protocol. The latency time associated with the round trip write request of an attribute protocol message was assessed, Figure 11. The data were processed using $\mathrm{Matlab}^{4}$ to plot the latency distribution. Evaluation was performed by measuring the time between the App (client) writing a remote FES configuration attribute on the FES device GATT server and the receipt of a write acknowledgement on the App. A total of 1501 Write operations were performed through the App and the latency was measured, with the BLE connection between the App and FES device pre-established. The evaluation was performed twice at Received signal strength (RSSI) of $-75 \mathrm{~dB}$ and $\sim-95 \mathrm{~dB}$.

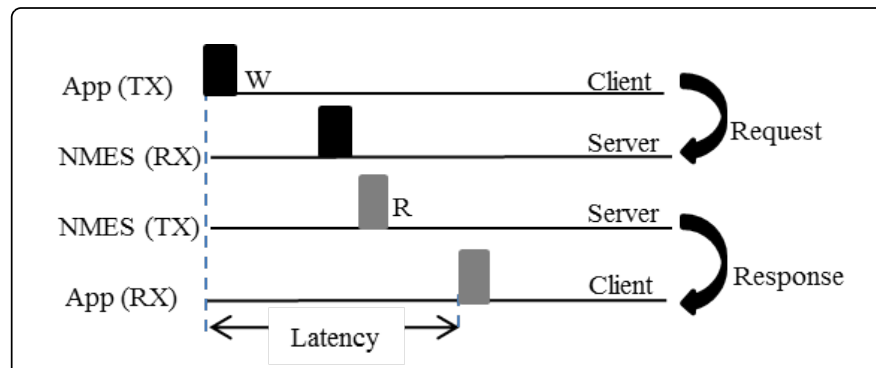

Figure 11: Request to response latency timing diagram.

\section{Usability}

The App was developed using an iterative User Centred Design approach as presented by Harte et al. Development of the App was carried out using an agile approach, with each iteration of the App tested on the target user population and detailed feedback sought in each case. Following feedback, changes were made to the App and the process repeated until an App with good user acceptance was achieved.

\section{Results}

Simulations of RCT Treatment generation and allocation were conducted using the App to evaluate the functionality and reliability of the processes. The maximum setting of 100 generated treatments and 1000 participants with 10 blocks of treatments was simulated; the average time to generate and store the Treatment Array and Treatment List was $\sim 28 \mathrm{~s}$.

Figure 12 shows the cumulative distribution of the latency time between the App writing a remote FES configuration attribute on the FES device GATT server and the receipt of a write acknowledgement by the App. At an RSSI of $-75 \mathrm{~dB}$ the latency time was $<15 \mathrm{~ms}$ for $78 \%$ and $<20 \mathrm{~ms}$ for $97 \%$ of the 1501 write operations. At an RSSI of $-95 \mathrm{~dB}$ the latency time was $<15 \mathrm{~ms}$ for $56 \%$ and $<20 \mathrm{~ms}$ for $93 \%$ of the 1501 write operations. The longest time for a response was $34 \mathrm{~ms}$ and in all cases zero packets were lost. 
Citation: Sweeney D, Corley GJ, Browne P, Burridge JH, Quinlan LR, Laighin ÓG (2016) Design of a Smartphone Application with Integrated Functional Electrical Stimulation (FES) Treatment Randomization and On-The-Fly Stimulus Parameter Adjustment for Streamlining the Clinical Evaluation of FES Protocols. J Health Med Inform 7: 220. doi:10.4172/2157-7420.1000220

Page 7 of 9

The results from the normal probability plot are presented in Figure 13. The $x$-axis data represent the distribution of the 720,000 generated Treatment Array frequencies. From observation of the normal probability plot it is evident that the processed data exhibit the standard "bell curve" or Gaussian distribution centered on a frequency of 1000 .

The results from the runs test with a significance level of 0.05 are given in Table 1 and support the hypothesis that the series of generated Treatment Arrays are in a random order

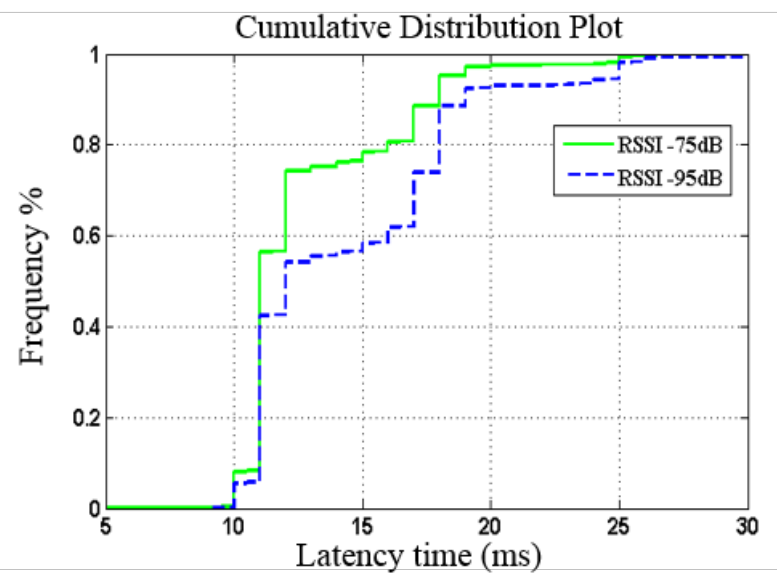

Figure 12: Latency time cumulative distribution plot.

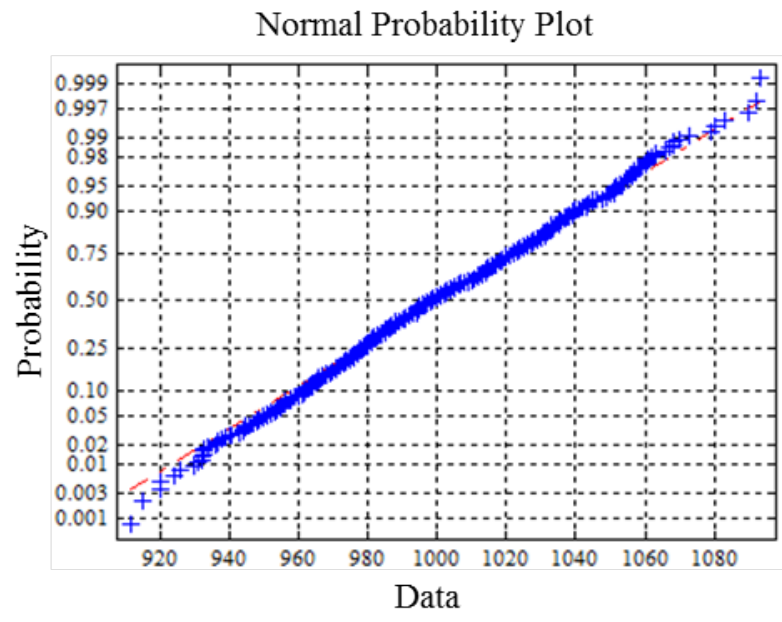

Figure 13: Randomization probability plot.

\begin{tabular}{|l|l|l|l|}
\hline \multirow{2}{*}{$\begin{array}{l}\text { Runs Test } \\
\text { Results }\end{array}$} & \multicolumn{3}{|l|}{ Sequence Length } \\
\cline { 2 - 4 } & 7200 & 720000 & 720000 \\
\hline P - value & 0.6253 & 0.6789 & 0.5704 \\
\hline Null hypothesis & True & True & True \\
\hline
\end{tabular}

Table 1: Runs test results for $7200,72000$.

\section{Conclusion}

In this paper, a smartphone App for the control of an FES device, which addresses some of the current challenges associated with the use of FES in research and clinical trial settings was presented. The App was designed to meet the requirements of clinicians and researchers working in the area of FES. The Apps demonstrates a capability to perform such functions as Treatment Generation, Allocation Concealment, Allocation Randomization, Blind Allocation Assignment, FES Device Configuration and electronic data logging. The App would thus serve to significantly streamline the implementation of FES RCTs by reducing extensive manual data collection, reducing the research staff required, removing the necessity for assistance of any third party during treatment allocation (Figure 14) and providing on-the-fly FES parameter adjustment. Results from the evaluation show good reliability and performance thereby supporting the implementation of blinded randomized control and temporal control of an FES device through a smartphone application.

It is anticipated that the use of smartphone technology, Bluetooth enable FES devices and the development of Apps will aid in the design of effective RCTs of FES systems by providing a portable and flexible platform that will reduce costs and increase the robustness and efficiency of these studies. The costs associated with developing smartphone applications for research purposes can be relatively low, with Android providing an open source platform for development and Apple requiring a yearly fee of $\$ 99$ to develop Apps for the Apple platform. For researchers with App development experience the majority of the costs are associated with the development time required to customize the App to meet the specific trial methodology requirements and to develop the Bluetooth communication protocol. As there is no specialized standard for FES devices within the IEEE 111073 Personal Health standard repository, in this study an in-house FES device was used with a proprietary GATT profile communication protocol. The development of a standard GATT profile for FES devices may enable greater interoperability among Bluetooth enabled FES devices and smartphone applications.

In addition to considering the cost of developing the App, concerns over usability and security may affect the acceptance of the technology. Researchers considering the development and use of similar Apps should consult with clinicians throughout the development stages to identify potential issues such as button size and interface layout. In this work the clinical team recommended, large buttons with tactile feedback be adopted and these features are embedded in the developed App.

Although our App demonstrated the ability to support the implementation of blind randomized control and temporal control of an FES device we acknowledge it is only developed for a single site trial. Further work may consider the development of a multi-site trial randomization App. Although such implementations will add additional cost to the App development and require additional security and infrastructure cost through the use of server and network provider resources, they may provide a cost effective alternative to existing methods of multi-site trial randomization services. 
Citation: Sweeney D, Corley GJ, Browne P, Burridge JH, Quinlan LR, Laighin ÓG (2016) Design of a Smartphone Application with Integrated Functional Electrical Stimulation (FES) Treatment Randomization and On-The-Fly Stimulus Parameter Adjustment for Streamlining the Clinical Evaluation of FES Protocols. J Health Med Inform 7: 220. doi:10.4172/2157-7420.1000220

Page 8 of 9

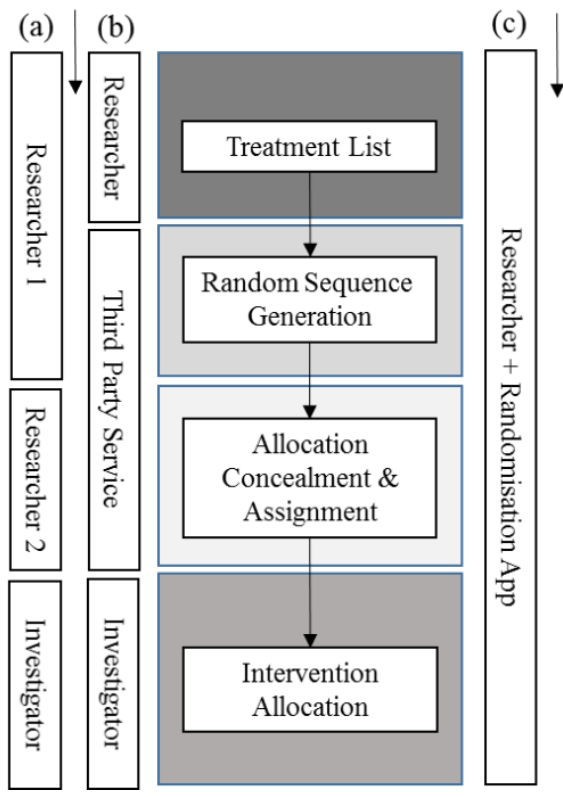

Figure 14: General RCT randomization procedure: (a) Without third party services, (b) With third party services, (c) Utilizing the randomisation App.

\section{Acknowledgement}

This work has been performed within the framework of the FP7 project REMPARK ICT-287677, which was funded by the European Commission.

\section{References}

1. Lyons GM, Sinkjær T, Burridge JH, Wilcox DJ (2002) A review of portable FES-based neural orthoses for the correction of drop foot. IEEE Transactions on Neural Systems and Rehabilitation Engineering 10: 260-279.

2. Malešević NM, Maneski LZ, Ilić V, Jorgovanović N, Bijelić G, et al. (2012) A multi-pad electrode based functional electrical stimulation system for restoration of grasp. Journal of Neuroengineering and Rehabilitation 9: 66.

3. Downey RJ, Bellman MJ, Kawai H, Gregory CM, Dixon WE (2015) Comparing the Induced Muscle Fatigue Between Asynchronous and Synchronous Electrical Stimulation in Able-Bodied and Spinal Cord Injured Populations. IEEE Trans Neural Syst Rehabil Eng 23: 964-972.

4. Barton S (2000) Which clinical studies provide the best evidence? The best RCT still trumps the best observational study. BMJ: British Medical Journal 321: 255-256.

5. Sibbald B, Roland M (1998) Understanding controlled trials. Why are randomised controlled trials important? BMJ: British Medical Journal 316: 201.

6. Altman DG, Bland JM (1999) How to randomize. BMJ: British Medical Journal 319: 703-704.

7. Suresh KP (2011) An overview of randomization techniques: an unbiased assessment of outcome in clinical research. Journal of Human Reproductive Sciences 4: 8-11.

8. Saghaei M (2004) Random allocation software for parallel group randomized trials. BMC Medical Research Methodology 4: 1-26.
9. Schulz KF, Grimes DA (2002) Allocation concealment in randomised trials: defending against deciphering. The Lancet 359: 614-618.

10. Herbison P, Hay SJ, Gillespie WJ (2011) Different methods of allocation to groups in randomized trials are associated with different levels of bias. A meta-epidemiological study. Journal of Clinical Epidemiology 64: 1070-1075.

11. Doig GS, Simpson F (2005) Randomization and allocation concealment: a practical guide for researchers. Journal of Critical Care 20: 187-191.

12. Viera AJ, Bangdiwala SI (2007) Eliminating bias in randomized controlled trials: importance of allocation concealment and masking. Fam Med 39: 132-137.

13. Hewitt C, Hahn S, Torgerson DJ, Watson J, Bland JM (2005) Adequacy and reporting of allocation concealment: review of recent trials published in four general medical journals. BMJ: British Medical Journal 330: 1057-1058.

14. Torgerson DJ, Roberts C (1999) Understanding controlled trials: Randomisation methods: concealment. BMJ: British Medical Journal 319: 375-376.

15. Clark L, Schmidt U, Tharmanathan P, Adamson J, Hewitt C, et al. (2013) Allocation concealment: a methodological review. Journal of evaluation in clinical practice 19: 708-712.

16. Beksinska ME, Joanis C, Smit JA, Pienaar J, Piaggio G (2013) Using scratch card technology for random allocation concealment in a clinical trial with a crossover design. Clinical Trials 10: 125-130.

17. Armijo OS, Saltaji H, da Costa BR, Fuentes J, Ha C, et al. (2015) What is the influence of randomisation sequence generation and allocation concealment on treatment effects of physical therapy trials? A metaepidemiological study. BMJ open 5: e008562.

18. Clark L, Schmidt U, Tharmanathan P, Adamson J, Hewitt C, et al. (2013) Poor reporting quality of key Randomization and Allocation Concealment details is still prevalent among published RCTs in 2011: a review. Journal of evaluation in clinical practice 19: 703-707.

19. Chen X, Zhai X, Wang X, Su J, Li M (2014) Methodological reporting quality of randomized controlled trials in three spine journals from 2010 to 2012. European Spine Journal 23: 1606-1611.

20. De J Guirro RR, de Oliveira GEC, de Sousa NT (2015) Sensory and Motor Thresholds of Transcutaneous Electrical Stimulation Are Influenced by Gender and Age. PM\&R 7: 42-47.

21. Dantas LO, Vieira A, Siqueira AL, Salvini TF, Durigan JL (2015) Comparison between the effects of 4 different electrical stimulation current waveforms on isometric knee extension torque and perceived discomfort in healthy women. Muscle and Nerve 51: 76-82.

22. Scott W, Adams C, Cyr S, Hanscom B, Hill K, et al. (2015) Electrically Elicited Muscle Torque: Comparison Between $2500 \mathrm{~Hz}$ Burst-Modulated Alternating Current and Monophasic Pulsed Current. J Ortho Sports Phys Ther 45: 1035-1041.

23. Wu D, Qian L, Zorowitz RD, Zhang L, Qu Y, et al. (2013) Effects on decreasing upper-limb poststroke muscle tone using transcranial direct current stimulation: a randomized sham-controlled study. Arch Phys Med Rehabil 94: 1-8.

24. Szecsi J, Fornusek C (2014) Comparison of torque and discomfort produced by sinusoidal and rectangular alternating current electrical stimulation in the quadriceps muscle at variable burst duty cycles. Am J Phys Med Rehabil 93: 146-159.

25. Popovic MR, Keller $\mathrm{T}$ (2005) Modular transcutaneous functional electrical stimulation system. Med Eng Phys 27: 81-92.

26. Broderick BJ, Kennedy C, Breen PP, Kearns SR, ÓLaighin G (2011) Patient tolerance of neuromuscular electrical stimulation (NMES) in the presence of orthopaedic implants. Med Eng Phys 33: 56-61.

27. Kortum P (2008) HCI Beyond the GUI: Design for Haptic, Speech, Olfactory, and Other Nontraditional Interfaces. Morgan Kaufmann Publishers Inc., San Francisco, CA, USA.

28. Batagelj V, Brandes U (2005) Efficient generation of large random networks. Physical Review E 71: 036113. 
Citation: Sweeney D, Corley GJ, Browne P, Burridge JH, Quinlan LR, Laighin ÓG (2016) Design of a Smartphone Application with Integrated Functional Electrical Stimulation (FES) Treatment Randomization and On-The-Fly Stimulus Parameter Adjustment for Streamlining the Clinical Evaluation of FES Protocols. J Health Med Inform 7: 220. doi:10.4172/2157-7420.1000220

Page 9 of 9

29. Wald A, Wolfowitz J (1940) On test of weather two samples are from the same population. The Annals of Mathematical Statistics 11: 494-520.

30. Harte RP, Glynn LG, Broderick BJ, Rodriguez-MA, Baker P, et al. (2014) Human centred design considerations for connected health devices for the older adult. Journal of Personalized Medicine 4: 245-281.
31. Harte R, Quinlan LR, Glynn L, Rodriguez-MA, Scharf T, et al. (2015) A Multi-Stage Human Factors and Comfort Assessment of Instrumented Insoles Designed for Use in a Connected Health Infrastructure. Journal of personalized medicine 5: 487-508. 